\title{
Blue Laser Imaging, Blue Light Imaging, and Linked Color Imaging for the Detection and Characterization of Colorectal Tumors
}

\author{
Naohisa Yoshida ${ }^{1}$, Osamu Dohi ${ }^{1}$, Ken Inoue $^{1}$, Ritsu Yasuda ${ }^{1}$, Takaaki Murakami ${ }^{1}$, Ryohei Hirose ${ }^{1}$, Ken Inoue $^{1}$, Yuji Naito ${ }^{1}$, \\ Yutaka Inada ${ }^{2}$, Kiyoshi Ogiso ${ }^{3}$, Yukiko Morinaga ${ }^{4}$, Mitsuo Kishimoto ${ }^{4}$, Rafiz Abdul Rani ${ }^{5}$, and Yoshito Itoh ${ }^{1}$ \\ ${ }^{1}$ Department of Molecular Gastroenterology and Hepatology, Graduate School of Medical Science, Kyoto Prefectural University of Medicine, \\ ${ }^{2}$ Department of Gastroenterology, Fukuchiyama City Hospital, Kyoto, ${ }^{3}$ Department of Gastroenterology, Osaka General Hospital of West Japan \\ Railway Company, Osaka, ${ }^{4}$ Department of Surgical Pathology, Graduate School of Medical Science, Kyoto Prefectural University of Medicine, \\ Kyoto, Japan, and ${ }^{5}$ Gastroenterology Unit, Faculty of Medicine, Universiti Teknologi MARA, Shah Alam, Malaysia
}

\begin{abstract}
A laser endoscopy system was developed in 2012. The system allows blue laser imaging (BLI), BLI-bright, and linked color imaging $(\mathrm{LCl})$ to be performed as modes of narrowband light observation; these modes have been reported to be useful for tumor detection and characterization. Furthermore, an innovative endoscopy system using four-light emitting diode (LED) multilight technology was released in 2016 to 2017 in some areas in which laser endoscopes have not been approved for use, including the United States and Europe. This system enables blue light imaging (this is also known as BLI) and LCI with an LED light source instead of a laser light source. Several reports have shown that these modes have improved tumor detection. In this paper, we review the efficacy of $\mathrm{BLI}$ and $\mathrm{LCl}$ with laser and LED endoscopes in tumor detection and characterization. (Gut Liver 2019;13:140-148)
\end{abstract}

Key Words: Colorectal neoplasms; Linked color imaging; Blue laser imaging; Blue light imaging

\section{INTRODUCTION}

According to the World Health Organization (WHO), colorectal cancer (CRC) was the third-most common type of cancer with regard to morbidity and had the fourth highest rate of mortality among all cancers in 2012. ${ }^{1}$ In Japan, there were 149,500 cases and 53,000 deaths among a population of 126 million in 2017, making CRC the most common type of cancer with regard to morbidity and the second-most common cause of cancer-related death-and the incidence is still increasing. ${ }^{2}$
Due to the aging of the population, the incidence of CRC and the number of colonoscopy procedures are expected to increase in the future. The effective tumor detection, accurate diagnosis, and treatment of tumors are important for reducing the rate of CRC death.

For these reasons, a light amplification by stimulated emission of radiation (LASER) endoscopic system (LASEREO; Fujifilm Co., Tokyo, Japan) was developed in 2012. ${ }^{3}$ The system allows for blue laser imaging (BLI), BLI-bright, and linked color imaging (LCI) to be performed as modes of narrow-band light observation; these modes have been reported to be useful for tumor detection and characterization. ${ }^{4-18}$ Furthermore, an innovative endoscopy system using four-light emitting diode (LED) multilight technology (Fujifilm Co.) was released in 2016 to 2017 for some areas in which LASER endoscopes have not been approved for use, including the United States and Europe. ${ }^{19}$ This system enables blue light imaging (this is also called as BLI) and LCI with an LED light source instead of a LASER light source.

In this paper, we review the efficacy of BLI and LCI with LASER and LED endoscopes in colorectal tumor detection and characterization.

\section{THE CHARACTERISTICS OF LASER AND LED ENDO- SCOPES}

The new endoscope system "LASERE0," which was developed by Fujifilm, uses a semiconductor LASER as a light source. It has a narrow-band light observation function without a customized optical filter. The LASEREO system consists of a light source (LL-7000), a processor (VP-7000), and a series of special

Correspondence to: Naohisa Yoshida (https://orcid.org/0000-0001-6167-9705)

Department of Molecular Gastroenterology and Hepatology, Graduate School of Medical Science, Kyoto Prefectural University of Medicine, 465 Kajii-cho, Kawaramachi-Hirokoji, Kamigyo-ku, Kyoto 602-8566, Japan

Tel: +81-75-251-5519, Fax: +81-75-251-0710, E-mail: naohisa@koto.kpu-m.ac.jp

Received on June 15, 2018. Revised on July 23, 2018. Accepted on July 30, 2018. Published online December 4, 2018

pISSN 1976-2283 eISSN 2005-1212 https://doi.org/10.5009/gnl18276

() This is an Open Access article distributed under the terms of the Creative Commons Attribution Non-Commercial License (http://creativecommons.org/licenses/by-nc/4.0) which permits unrestricted non-commercial use, distribution, and reproduction in any medium, provided the original work is properly cited. 
scopes. The LL-7000 light source has two types of lasers with wavelengths of 410 and $450 \mathrm{~nm}$ (Fig. 1) ${ }^{4,5}$ and peak wavelength ranges of $410 \pm 10$ and $450 \pm 10 \mathrm{~nm}$, respectively. In addition, both bandwidths are less than approximately $2 \mathrm{~nm}$. In contrast, the wavelengths of narrow band imaging (NBI; Olympus, Tokyo, Japan) with a xenon lamp are $415 \mathrm{~nm}$ and $540 \mathrm{~nm}$ and the bandwidth of it is $30 \mathrm{~nm}$. ${ }^{4}$ The irradiation of phosphor by the 450-nm wavelength LASER achieves fluorescent light. The combination of strong LASER light with a 450-nm wavelength LASER and fluorescent light provides an enough degree of illumination for white-light imaging (WLI). The 410-nm wavelength LASER is used for BLI and functions as narrow-band light.

BLI light is made from the combination of the strong LASER light with a wavelength of $410 \mathrm{~nm}$, weak LASER light with a wavelength of $450 \mathrm{~nm}$, and fluorescent light. BLI light is useful for acquiring information about the mucosal surface, such as the patterns of surface blood vessels and structures. The use of LASERs with a specific wavelength and precisely regulated light power allows the blood vessels to be observed in detail and makes the surface patterns clear. The "BLI-bright" mode is generated by the appropriate combination of white light and BLI light, which is controlled by adjusting the power of the two LASERs. This mode is brighter than the BLI mode and is expected to be useful for tumor detection. LCI can also be performed using the light balance of similar power to BLI-bright and is useful for tumor detection. LCI makes lesions more reddish and the surrounding mucosa more whitish, creating good contrast for

\section{LASER endoscope}

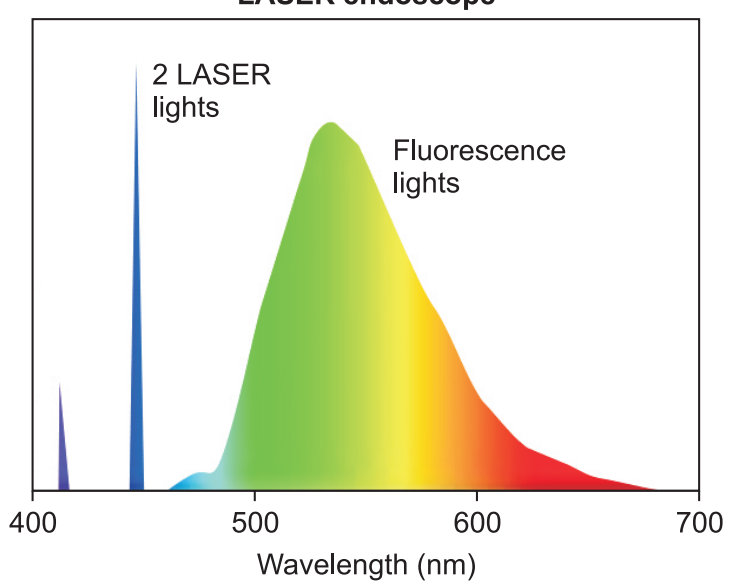

LED endoscope



Fig. 1. Characteristics of laser and light emitting diode (LED) endoscopy. LASER, light amplification by stimulated emission of radiation.

Table 1. Reports on BLI and Laser Imaging with a Laser Endoscope for Tumor Characteristics

\begin{tabular}{|c|c|c|c|c|c|c|c|c|}
\hline Author & System & Magnification & $\begin{array}{l}\text { No. of } \\
\text { cases }\end{array}$ & Subjects & $\begin{array}{c}\text { Overall } \\
\text { accuracy } \\
(\%)\end{array}$ & $\begin{array}{l}\text { Accuracy for } \\
\text { differentiating } \\
\text { between } \\
\text { neoplastic and } \\
\text { non-neoplastic } \\
(\%)\end{array}$ & $\begin{array}{l}\text { Sensitivity } \\
\text { of T1b } \\
\text { in invasive } \\
\text { BLI mag- } \\
\text { nification } \\
\text { patterns } \\
(\%)\end{array}$ & $\begin{array}{c}\text { Specificity } \\
\text { of T1b } \\
\text { in invasive } \\
\text { BLI mag- } \\
\text { nification } \\
\text { patterns } \\
(\%)\end{array}$ \\
\hline $\begin{array}{l}\text { Yoshida } \\
\text { et al. }\end{array}$ & BLI & Yes & 104 & Neoplastic lesions & 74.0 & NA & 37.5 & 100.0 \\
\hline $\begin{array}{l}\text { Yoshida } \\
\text { et al. } .^{5}\end{array}$ & BLI & Yes & 314 & $\begin{array}{c}\text { Neoplastic and non- } \\
\text { neoplastic lesions }\end{array}$ & 84.3 & 85.0 & 64.3 & 99.3 \\
\hline $\begin{array}{l}\text { Yoshida } \\
\text { et al. }\end{array}$ & BLI & No & 125 & $\begin{array}{c}\text { Neoplastic and non- } \\
\text { neoplastic lesions }\end{array}$ & NA & 95.2 & NA & NA \\
\hline $\begin{array}{l}\text { Nakano } \\
\text { et al. }\end{array}$ & BLI & Yes & 748 & $\begin{array}{c}\text { Neoplastic and non- } \\
\text { neoplastic lesions }\end{array}$ & 89.3 & 98.4 & 40.0 & 100.0 \\
\hline $\begin{array}{l}\text { Wu } \\
\text { et al. }\end{array}$ & LCI & No & 94 & $\begin{array}{l}\text { Neoplastic and non- } \\
\text { neoplastic lesionss }\end{array}$ & 91.5 & 91.5 & 100.0 & 99.0 \\
\hline $\begin{array}{l}\text { Suzuki } \\
\text { et al. }\end{array}$ & $\begin{array}{c}\mathrm{LCI}+ \\
\text { crystal violet }\end{array}$ & Yes & 3 & Tis & 100.0 & NA & NA & NA \\
\hline
\end{tabular}

BLI, blue laser imaging; LCI, linked color imaging; NA, not available. 
detecting tumors.

On the other hand, high-intensity illumination based on 4-LED Multi Light technology (BL-7000) provides high-quality images with white light and new observation modes of BLI and LCI. The system has four types of light: blue-violet, blue, green, and red (Fig. 1). ${ }^{19}$

\section{COLORECTAL TUMOR CHARACTERIZATION}

NBI and pit pattern observation are the gold standard for tumor characterization. ${ }^{20,21}$ BLI magnification is also regularly used to this end as well. This enables us to clearly observe the surface structures and vessel patterns. ${ }^{4,5}$ Some reports have shown the efficacy of BLI magnification (Table 1). ${ }^{4-6}$ Our previous study compared BLI and NBI. Endoscopic images of surface structures and vessel patterns obtained using BLI magnification were slightly different from those obtained with NBI magnification (Fig. 2). ${ }^{4}$ In detail, 104 colorectal neoplasms were examined with both BLI and NBI magnification. The Hiroshima classification was an NBI classification and was used to assess the surface structures and vessel patterns observed with NBI magnification in that study. ${ }^{22}$ The diagnostic accuracy of BLI magnification in the NBI classification was $74.0 \%$ (77/104), which was similar to that of NBI magnification (77.8\%). The rate of consistency between BLI and NBI magnification in the NBI classification was $74.0 \%$. Another study from our group showed that the diagnostic accuracy of BLI magnification was 84.3\% among 314 polyps (hyperplastic polyps and sessile serrated polyps [SSP], $\mathrm{n}=41$; low-grade adenoma, $n=168$; high-grade adenoma, $n=80$; 1 cancer, $n=25$ ) using the Hiroshima classification. ${ }^{5}$ The accuracy of differentiation between non-neoplastic and neoplastic lesions was 99.3\% (312/314), while that between adenomatous and cancerous lesions was $85.0 \%$ (232/273). The diagnostic accuracy for polyps $<20 \mathrm{~mm}$ in diameter was better than that for polyps $\geq 20 \mathrm{~mm}$ in diameter $(92.1 \%$ vs $72.5 \%, \mathrm{p}<0.001)$. The diagnostic accuracy with regard to the morphology was significantly lower for superficial polyps of $\geq 20 \mathrm{~mm}$ in diameter than for superficial polyps of $<20 \mathrm{~mm}$ in diameter (70.0\% vs $82.9 \%$, p=0.03). In addition, the diagnostic accuracy of BLI without magnification for differentiating between neoplastic and non-neoplastic polyps $<10 \mathrm{~mm}$ in diameter was 95.2\%, which was greater than that of white light (83.2\%).

The diagnostic accuracy of BLI magnification and pit pattern observation was compared in a previously published study. ${ }^{6}$ The diagnostic accuracy for the differentiation of neoplastic from non-neoplastic lesions was 98.4\% with BLI and 98.7\% with pit pattern observation. In addition, the diagnostic accuracy of BLI magnification for $\mathrm{T} 1 \mathrm{~b}$ cancers was $89.5 \%$, while that of pit pattern observation was $92.1 \%$. The study further suggested that pit pattern observation should be performed for lesions with severely irregular surface structures and vessel patterns on BLI in order to improve the diagnostic accuracy.

Recently, the Japan NBI Expert Team (JNET) classification has been reported to be useful for differentiating between ad-
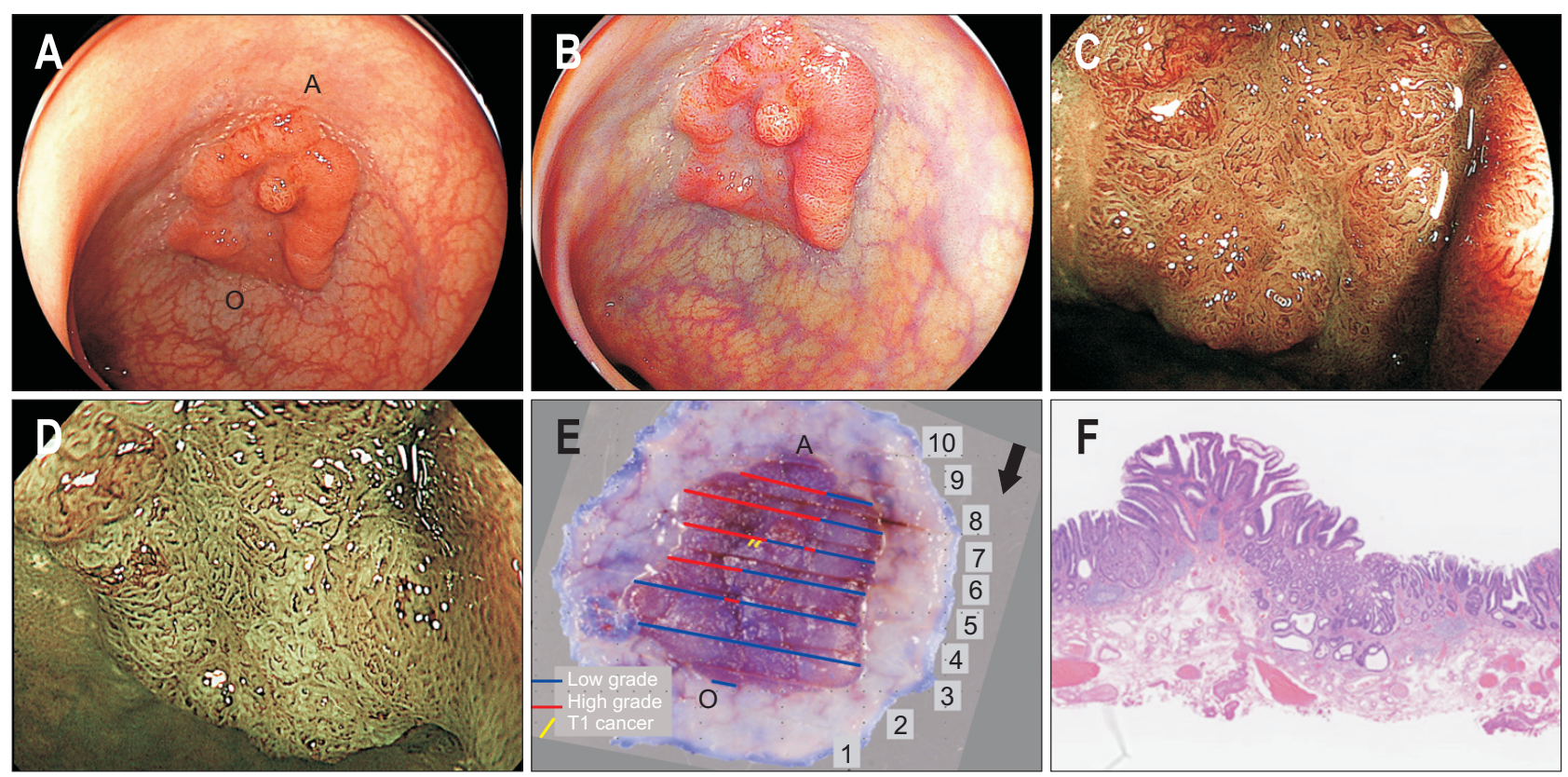

Fig. 2. T1 cancer observed by linked color imaging (LCI) and blue laser imaging (BLI) with a laser endoscope. (A) A nonpolypoid polyp 20 mm in size with depression. (B) LCI presented the lesion as reddish and increased polyp visibility. (C) BLI magnification revealed irregular surface patterns and vessel patterns (JNET type 2B). (D) Narrow band imaging (NBI) magnification revealed irregular surface patterns and vessel patterns, similar to BLI magnification. (E) Mapping of the resected specimen. T1 cancer (submucosal invasion length, $500 \mu \mathrm{m}$ ) with adenoma. Direction for an endoscopic observation (arrow). (F) Histological findings of submucosal invasion (H\&E, $\times 100)$.

0, oral side; A, anal side; JNET, Japan NBI Expert Team. 


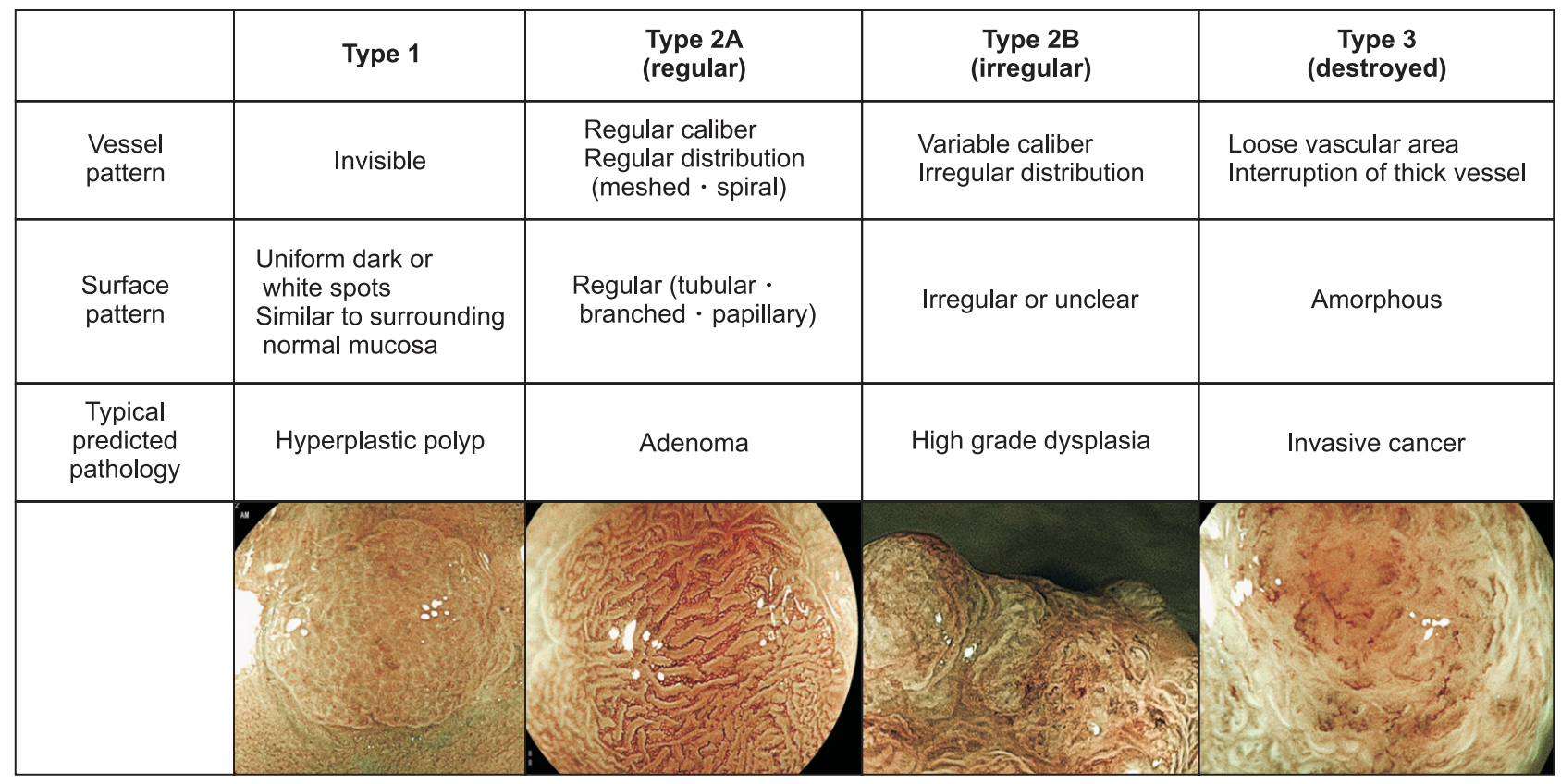

Fig. 3. JNET classifications.

JNET, Japan narrow band imaging (NBI) Expert Team.
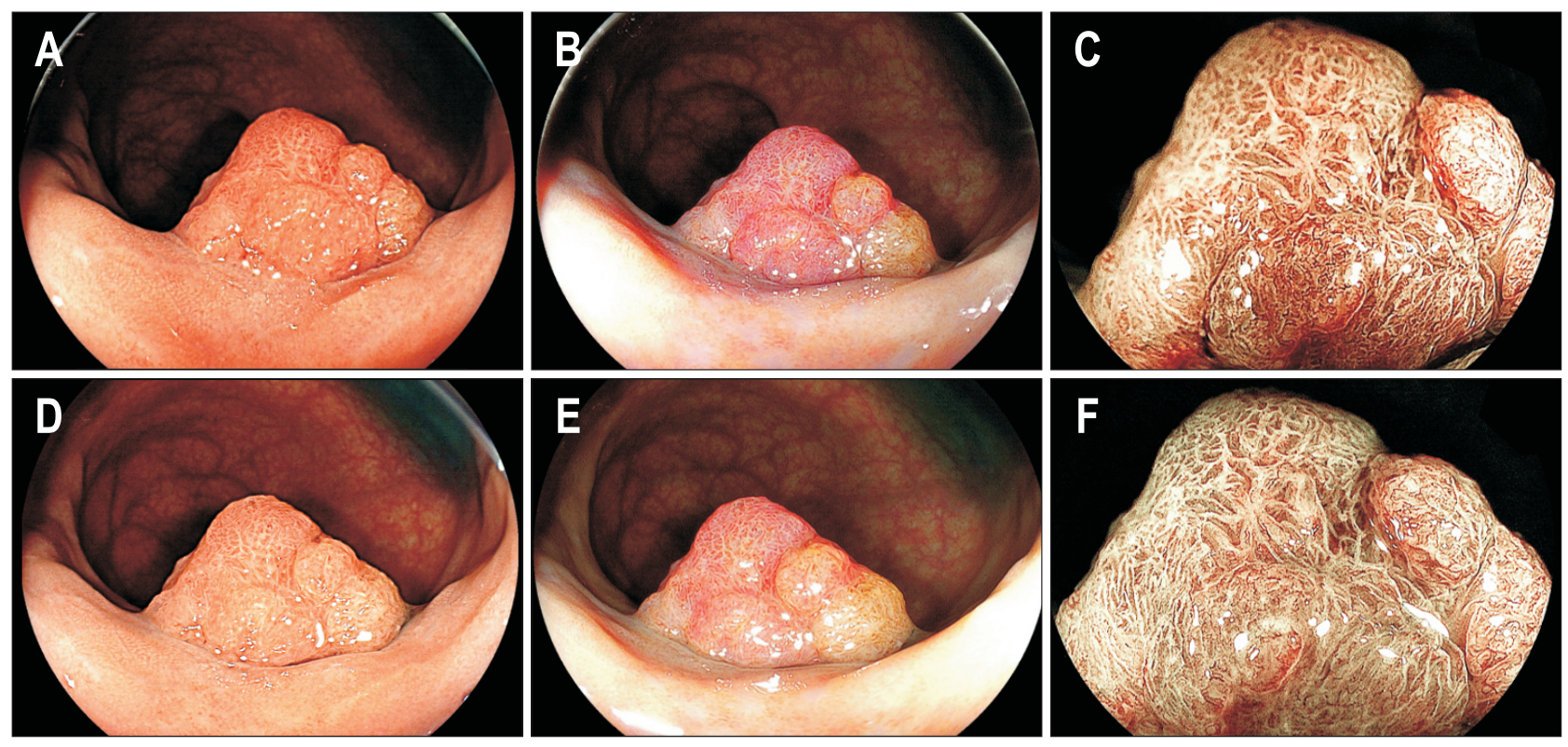

Fig. 4. A comparison of laser and light emitting diode (LED) endoscopes in the detection of high-grade rectal dysplasia. (A) White-light imaging (WLI) with a laser endoscope. (B) Linked color imaging (LCI) with a laser endoscope. (C) Blue laser imaging (BLI) with a laser endoscope (JNET classification: type 2B). (D) WLI with an LED endoscope. (E) LCI with an LED endoscope. (F) Blue light imaging (BLI) with an LED endoscope (JNET classification: type $2 \mathrm{~B})$.

JNET, Japan narrow band imaging (NBI) Expert Team.

enoma, high-grade dysplasia, and T1b cancer (Fig. 3). ${ }^{20}$ While it was originally performed with NBI, the JNET classification can also be performed with BLI; we previously demonstrated high consistency in the findings of NBI and BLI magnification as described above. ${ }^{4}$

On the other hand, no studies have reported the efficacy of
BLI with LED endoscopes with or without magnification for tumor characterization. In our experience, LEDs and LASERs are almost similar; however, LED light is generally a little brighter than LASER light. We believe that the various classifications used in BLI with a LASER endoscope can also be used in BLI with an LED endoscope (Fig. 4). In a BASIC study, a European 
group proposed that BLI with an LED light source could be used to differentiate non-neoplastic lesions from neoplastic ones based on the surface characteristics (presence of mucus, regular/ irregular, presence of depression), pit pattern (featureless, type, round with or without dark spots, homogenous distribution or heterogenous one), and vessel characteristics (presence, lacy, pericryptal, irregular). ${ }^{19}$ The accuracy of this classification is currently being examined. Regarding SSP, two previously reported endoscopic findings detected by NBI magnification can be used: thick and branched vessels and expanded crypt opening (Fig. 5). ${ }^{23}$ A unique method of observation combining LCI with a LASER light source and crystal violet dyeing was reportedly applied in a small number of cases (Table 1). ${ }^{7}$ This approach improved the contrast of the pit pattern, allowing the endoscopist to determine whether or not it had been destroyed (Fig. 6). Another unique study from China showed that LCI with a LASER light source enabled NICE classification. ${ }^{11}$
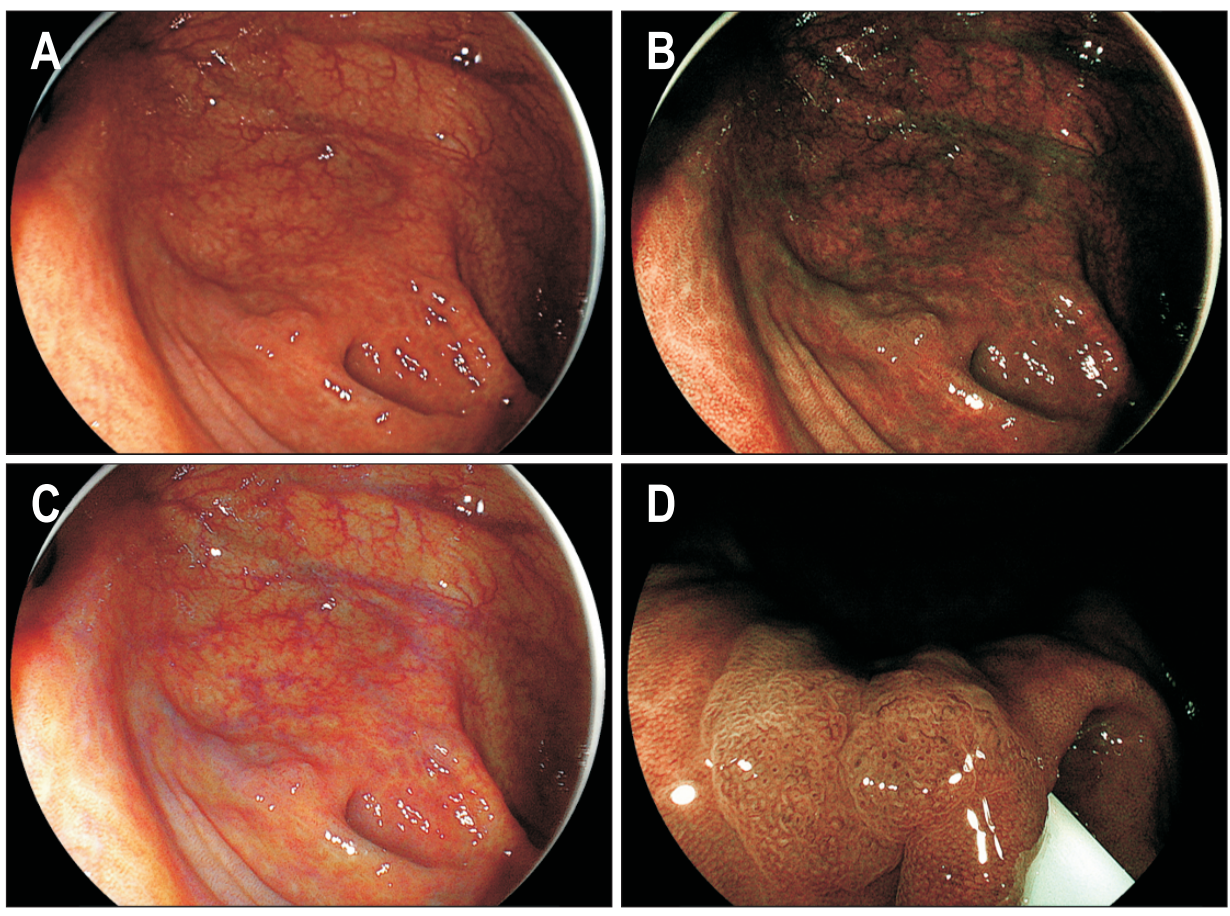

Fig. 5. A sessile, serrated polyp 10 $\mathrm{mm}$ in size in the cecum viewed with a laser endoscope. (A) Whitelight imaging. (B) Blue laser imaging (BLI)-bright. (C) Linked color imaging, (D) BLI magnification showing thick and branched vessels and an enlarged crypt opening.
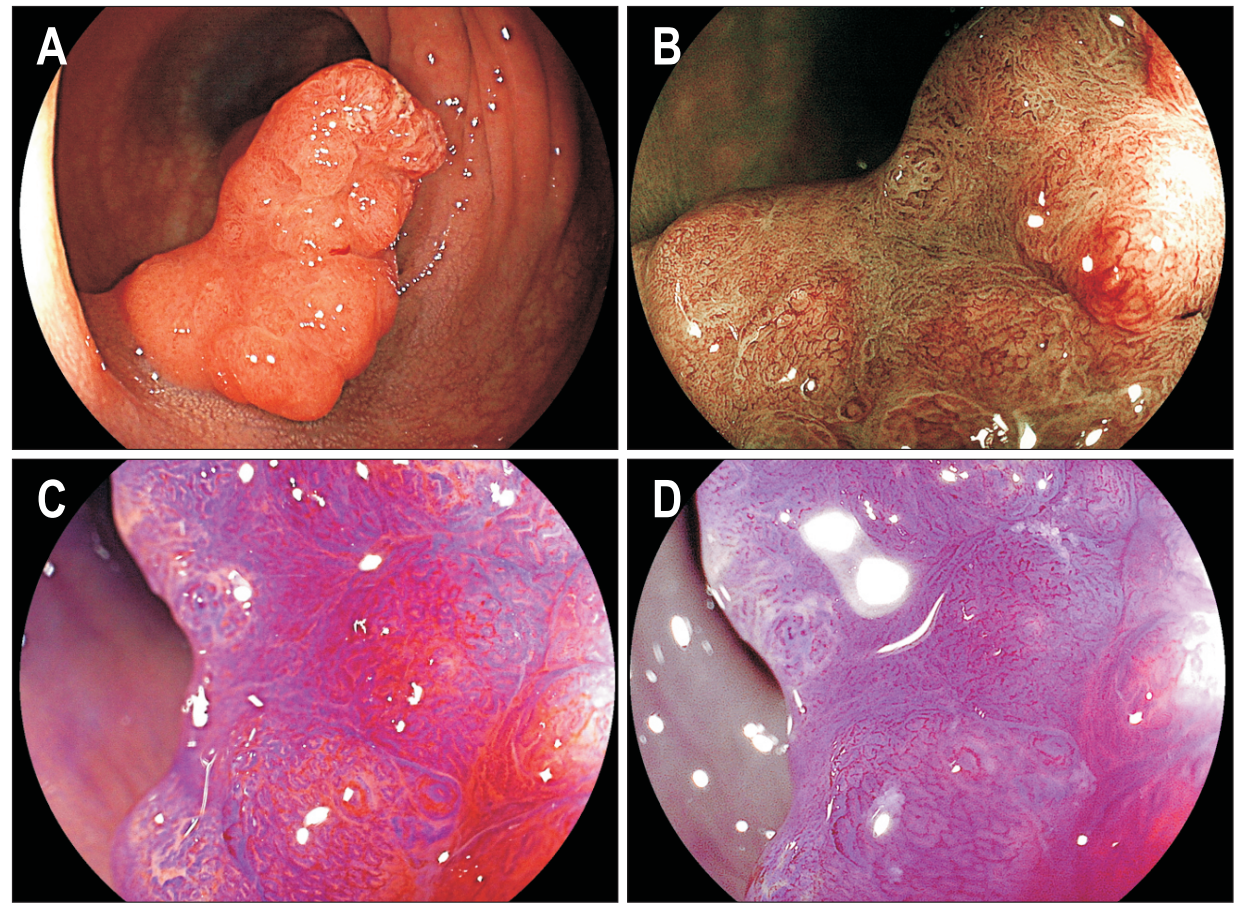

Fig. 6. A polypoid tumor $25 \mathrm{~mm}$ in size in the sigmoid colon, highgrade dysplasia, as viewed with a laser endoscope. (A) White-light imaging showing a polypoid tumor with slight depression (possible T1 cancer). (B) Blue laser imaging magnification showing a severely irregular surface pattern. The margin of the surface pattern was unclear. (C) Pit pattern observation with crystal violet staining showing a severely irregular pattern; however, some of the area was not stained well. (D) Linked color imaging with crystal violet staining clearly showed an irregular pit pattern. 


\section{COLORECTAL TUMOR DETECTION}

The removal of adenomas by colonoscopy has been proven to reduce the incidence of CRC and CRC-related death. ${ }^{24-26}$ However, it was reported that $20 \%$ to $27 \%$ of polyps were missed by WLI. ${ }^{27,28}$ Various factors have been reported to be associated with an increased risk of missing polyps, including a flat morphology, small size, presence in the ascending colon, male sex, multiple polyps at the time of first colonoscopy, and a history of polyps. $^{29,30}$

BLI-bright is brighter than BLI and is expected to show improved rates of tumor detection. For colorectal tumors, BLIbright makes a neoplastic lesion appear brownish in color, facilitating their detection. Our previous study on polyp visibility was performed with short movies of polyps recorded with BLIbright and WLI and an original polyp visibility scoring system (score 4, excellent visibility; score 3, good; score 2, fair; score 1, poor). ${ }^{8}$ In the movies of 100 whole neoplastic and non-neoplastic polyps, BLI-bright achieved higher scores than WLI (experts: $3.10 \pm 0.95$ vs $2.90 \pm 1.09, \mathrm{p}=0.00013$; non-experts: $3.04 \pm 0.94$ vs $2.78 \pm 1.03, \mathrm{p}<0.0001)$ (Fig. 7). In addition, a subgroup analysis of experts only showed that the mean visibility score of BLIbright mode was significantly higher than that of WLI for flat polyps, neoplastic polyps, and polyps located in the left-sided colon and rectum.

There have been some randomized controlled trials (RCTs) for detection using BLI (Table 2). Our previous multicenter RCT showed that BLI-bright $(n=489)$ resulted in a greater mean number of adenomatous polyps detected per patient than WLI $(\mathrm{n}=474)(1.27 \pm 1.73$ vs $1.01 \pm 1.36, \mathrm{p}=0.008)$ and also improved the mean total number of polyps per patient compared with WLI $(1.84 \pm 2.09$ vs $1.43 \pm 1.64, \mathrm{p}=0.001){ }^{9}$ However, BLI-bright required a longer observation times than WLI $(9.5 \pm 3.8$ minutes vs $8.4 \pm 2.9$ minutes, $\mathrm{p}<0.001)$. Another RCT in which patients
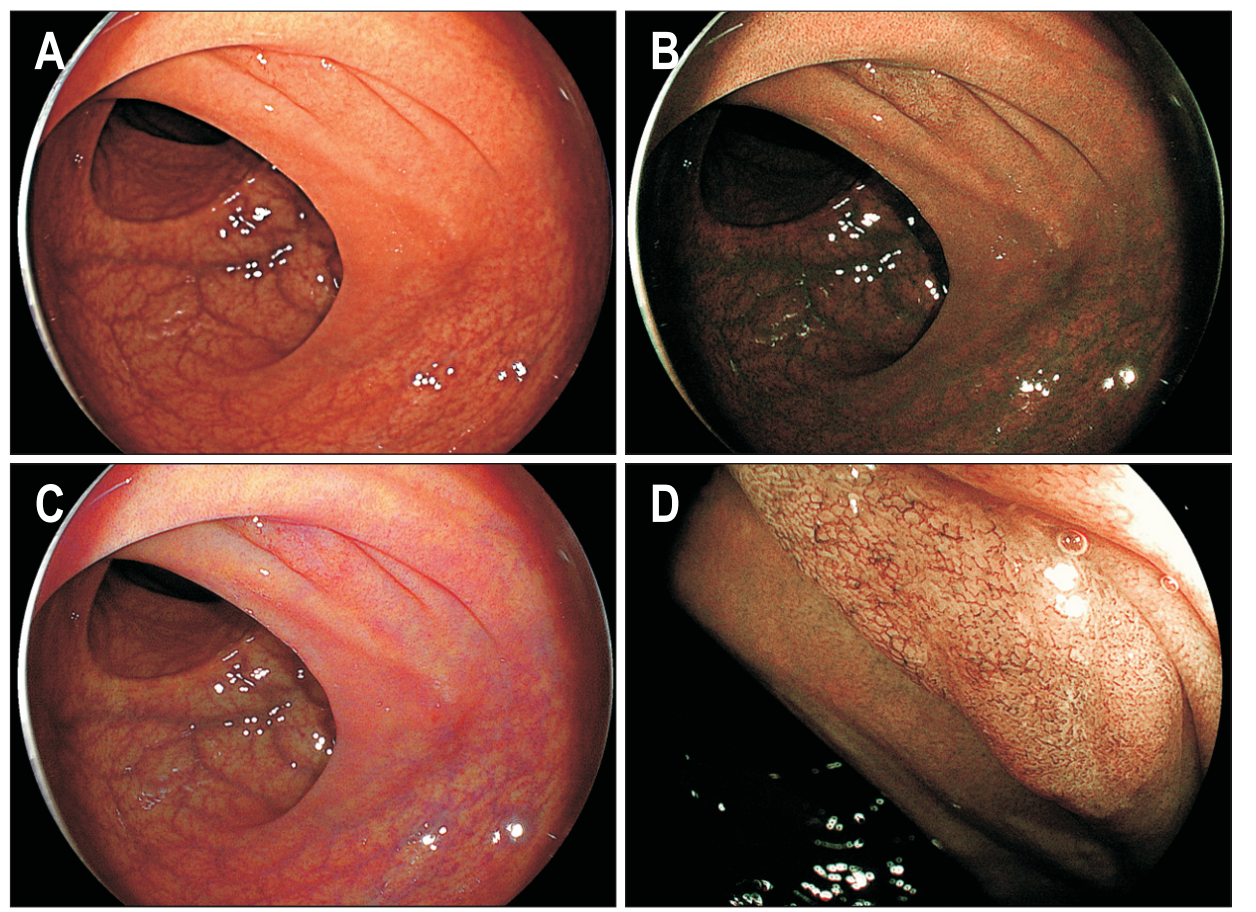

Fig. 7. A nonpolypoid tumor $12 \mathrm{~mm}$ in size in the rectum, high-grade dysplasia, as viewed with a laser endoscope. (A) White-light imaging showing a nonpolypoid tumor. The margin was not clear (polyp visibility score: 2). (B, C) Blue laser imaging (BLI)-bright and Linked color imaging performed with a laser endoscope showing a clear tumor margin (polyp visibility score: 4). (D) BLI magnification showing irregular surface patterns and vessel patterns (JNET classification: type 2B). JNET, Japan narrow band imaging (NBI) Expert Team.

Table 2. Reports on BLI and Laser Imaging with a Laser Endoscope for Tumor Detection

\begin{tabular}{|c|c|c|c|c|c|}
\hline Author & System & Setting & No. of cases & Methods & Efficacy \\
\hline Ikematsu et al. ${ }^{9}$ & BLI with LASER & RCT & 963 & ADR from the cecum to hepatic flexure & Positive \\
\hline Shimoda et al. ${ }^{10}$ & BLI with LASER & RCT & 127 & ADR in tandem endoscope for the right sided colon & Positive \\
\hline Min et al. ${ }^{15}$ & LCI with LASER & RCT & 141 & $\begin{array}{l}\text { PDR and ADR in tandem endoscope in tandem endo- } \\
\text { scope for whole the colorectum }\end{array}$ & Positive \\
\hline Fujimoto et al. ${ }^{16}$ & LCI with LASER & RCT & 44 & $\begin{array}{l}\text { SSP detection in tandem endoscope from the cecum to } \\
\text { splenic flexure }\end{array}$ & Positive \\
\hline Paggi et al. ${ }^{17}$ & LCI with LED & RCT & 600 & ADR in tandem endoscope for the right sided colon & Positive \\
\hline Yoshida et al. ${ }^{18}$ & LCI with LASER & Parallel & 130 & Additional 30 seconds observation to the right sided colon & Positive \\
\hline
\end{tabular}

BLI, blue laser imaging; LASER, light amplification by stimulated emission of radiation; RCT, randomized control study; ADR, adenoma detection rate; LCI, linked color imaging; PDR, polyp detection rate; SSP, sessile serrated polyp. 
were randomized to one of two tandem colonoscopy groups (BLI followed by WLI or WLI followed by BLI) showed that the polyp miss rate in the BLI-WLI group was $1.6 \%$, which was significantly lower than that in the WLI-BLI group $(10.0 \%, \mathrm{p}=0.001){ }^{10}$

BLI-bright observation is not widely accepted; however, there have been some positive reports, as described above. There are two major limitations regarding the use of BLI-bright. First, the residual liquid becomes reddish, which disturbs the endoscopic view. Second, the endoscopic view of BLI-bright is darker than with WLI, and NBI has the same problems. LCI has the potential to resolve these problems because it is brighter than BLI and BLI-bright and because the residual liquid becomes yellowish with LCI (Fig. 8). We previously reported that LCI improved the polyp visibility score using endoscopic movies. ${ }^{12}$ Among the LCI, BLI-bright, and WLI movies of 101 colorectal polyps, the mean polyp visibility scores of LCI $(2.86 \pm 1.08)$ were higher than
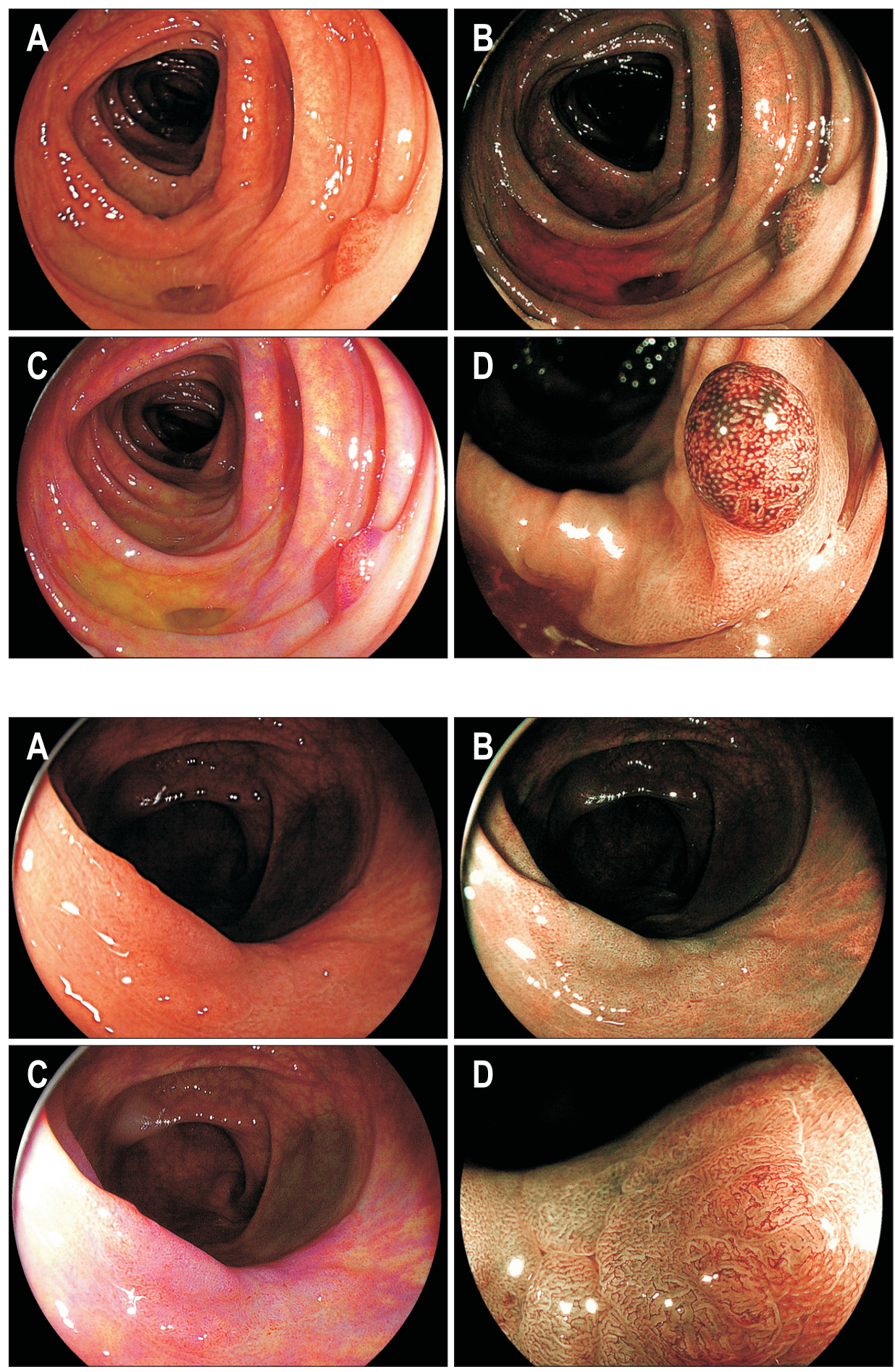

Fig. 8. A polypoid tumor $4 \mathrm{~mm}$ in size in the ascending colon, lowgrade adenoma, as viewed with a laser endoscope. (A) White-light imaging (WLI) showing a polyp and a small amount of residual liquid. (B) Blue laser imaging (BLI)-bright showing a polyp and reddish residual liquid; this endoscopic view was darker than that of WLI. (C) Linked color imaging showing a polyp and yellowish residual liquid; this endoscopic view was brighter than that of BLI-bright. (D) BLI magnification showed a regular surface patterns and vessel patterns.

Fig. 9. A nonpolypoid tumor 30 $\mathrm{mm}$ in size, high-grade dysplasia, as viewed with a laser endoscope. (A) WLI (polyp visibility score, 3; color difference value, 9.8). (B) BLI-bright (polyp visibility score, 4; color difference value, 18.0). (C) LCI (polyp visibility score, 4 ; color difference value, 22.0). (D) BLI magnification showing an irregular pattern (JNET classification: type 2B).

WLI, white-light imaging; BLI, blue laser imaging; LCI, linked color imaging; JNET, Japan narrow band imaging (NBI) Expert Team. 
those of WLI $(2.54 \pm 1.15, \mathrm{p}<0.001)$ and BLI-bright $(2.73 \pm 1.47$, $\mathrm{p}<0.001)$. In addition, LCI resulted in a reduced number of polyps that showed poor polyp visibility scores (scores of 1 or 2 ) compared with WLI (experts: $35.6 \%$ vs $49.6 \%$, p<0.015; nonexperts: $33.6 \%$ vs $50.5 \%, p=0.046)$. Furthermore, this study showed the efficacy of LCI for observing non-polypoid tumors and SSPs. ${ }^{12}$ Another study that we performed using endoscopic images showed the efficacy of LCI in the observation of diminutive polyps $\left(<5 \mathrm{~mm}\right.$ in size).$^{13}$ In that study, the color difference values between the tumor and the surrounding mucosa were calculated among endoscopic images of 54 colorectal polyps obtained by WLI and LCI in order to evaluate polyp visibility. The color difference value is thought to be an objective indicator of the polyp visibility. That study showed that LCI improved the color difference values more than WLI $(33.6 \pm 13.9$ vs $20.7 \pm 13.6$, $\mathrm{p}<0.001$ ) (Fig. 9). A similar study using polyp visibility scores showed that LCI improved the endoscopic visualization of nongranular colorectal lesions and also demonstrated the efficacy of LCI in the observation of SSPs. ${ }^{14}$

Several studies have shown the usefulness of LCI in polyp detection (Table 2). A Chinese RCT proved that LCI increased the adenoma detection rate (ADR) (LCI vs WLI, 37\% vs 28\%; 95\% confidence interval, $2.3 \%$ to $19.4 \%$ ) (Table 2). ${ }^{15}$ That same study also revealed that LCI resulted in significantly improved rates of adenoma and SSP detection compared with WLI (91\% vs 73\%, p<0.001). A Japanese RCT of LCI showed that the polyp detection rate of 6 non-expert endoscopists $(92.3 \% \pm 2.9 \%)$ was significantly higher than with WLI $(72.7 \% \pm 11.5 \%, \mathrm{p}<0.01)$ for observations from the cecum to splenic flexure. ${ }^{16}$ Furthermore, in a tandem endoscopy setting, the SSP detection rate of the LCI group $(21.6 \%, 8 / 37)$ was significantly higher than that of the LCI-WLI group (3.2\%, 1/31; p=0.02). A European RCT of LCI with LED showed a significant difference in the adenoma miss rate in the right sided colon (cecum and ascending colon) between WLI and LCI (30.6\% vs 11.8\%, p<0.001). ${ }^{17}$ Furthermore, in our previous study, an additional 30-second observation with LCI after WLI observation was found to significantly improve the overall adenoma and SSP numbers compared with a second observation with WLI after initial WLI. ${ }^{18}$ In that study the cecum and ascending colon were observed with WLI first in all cases, after which the colonoscope was inserted again, and the cecum and ascending colon were observed for an additional 30 seconds using either LCI or WLI. In this additional 30-second observation, the cecum and ascending colon were sufficiently insufflated and observed from a distant view. The overall adenoma and SSP numbers in the first and second observations of the LCI and WLI groups were 48 and 36, respectively ( $p=0.02$ ). In addition, in the LCI group, the number of adenomas and SSPs in the first and second observations were significantly higher than in the first observation (48 vs 35, p=0.017). However, one limitation associated with LCI involved issues with halation in the endoscopic view due to the high brightness, which might lead to missed polyps.

\section{CONCLUSIONS}

BLI with or without magnification and LCI without magnification with a LASER endoscope are more effective for polyp characterization than other approaches. BLI with a LASER endoscope and LCI with a LASER or LED endoscope are particularly useful for polyp detection. The BLI and LCI images obtained with a LASER endoscopy were considered to be almost similar to those obtained with an LED endoscope.

\section{CONFLICTS OF INTEREST}

No potential conflict of interest relevant to this article was reported.

\section{REFERENCES}

1. Hamilton SR, Aaltonen LA. World Health Organization classification of tumors: pathology and genetics of tumours of the digestive system. Lyon: IARC Press, 2010.

2. Cancer Information Service by National Cancer Center. Cancer statics in JAPAN '16 [Internet]. National Cancer Center [cited 2018 Sep 13]. Available from: https://ganjoho.jp/en/professional/statistics/bronchure/2016_en.html.

3. Osawa H, Yamamoto H. Present and future status of flexible spectral imaging color enhancement and blue laser imaging technology. Dig Endosc 2014;26 Suppl 1:105-115.

4. Yoshida N, Hisabe T, Inada Y, et al. The ability of a novel blue laser imaging system for the diagnosis of invasion depth of colorectal neoplasms. J Gastroenterol 2014;49:73-80.

5. Yoshida N, Yagi N, Inada Y, et al. Ability of a novel blue laser imaging system for the diagnosis of colorectal polyps. Dig Endosc 2014;26:250-258

6. Nakano A, Hirooka Y, Yamamura T, et al. Comparison of the diagnostic ability of blue laser imaging magnification versus pit pattern analysis for colorectal polyps. Endosc Int Open 2017;5:E224E231.

7. Suzuki T, Hara T, Kitagawa Y, Yamaguchi T. Magnified endoscopic observation of early colorectal cancer by linked color imaging with crystal violet staining (with video). Gastrointest Endosc 2016;84:726-729.

8. Yoshida N, Hisabe T, Hirose R, et al. Improvement in the visibility of colorectal polyps by using blue laser imaging (with video). Gastrointest Endosc 2015;82:542-549.

9. Ikematsu H, Sakamoto T, Togashi K, et al. Detectability of colorectal neoplastic lesions using a novel endoscopic system with blue laser imaging: a multicenter randomized controlled trial. Gastrointest Endosc 2017;86:386-394.

10. Shimoda R, Sakata Y, Fujise T, et al. The adenoma miss rate of blue-laser imaging vs. white-light imaging during colonoscopy: a 
randomized tandem trial. Endoscopy 2017;49:186-190.

11. Wu CH, Chen TH, Hsu CM, et al. Linked-color imaging combined with the NICE classification system for optical diagnosis of colon polyps: new image-enhanced endoscopic technology for pathological prediction. Ther Clin Risk Manag 2017;13:1317-1321.

12. Yoshida N, Naito Y, Murakami T, et al. Linked color imaging improves the visibility of colorectal polyps: a video study. Endosc Int Open 2017;5:E518-E525.

13. Yoshida N, Naito Y, Yasuda R, et al. Linked color imaging improves the visibility of various featured colorectal polyps in an endoscopist's visibility and color difference value. Int J Colorectal Dis 2017;32:1253-1260.

14. Suzuki T, Hara T, Kitagawa Y, et al. Linked-color imaging improves endoscopic visibility of colorectal nongranular flat lesions. Gastrointest Endosc 2017;86:692-697.

15. Min M, Deng P, Zhang W, Sun X, Liu Y, Nong B. Comparison of linked color imaging and white-light colonoscopy for detection of colorectal polyps: a multicenter, randomized, crossover trial. Gastrointest Endosc 2017;86:724-730.

16. Fujimoto D, Muguruma N, Okamoto K, et al. Linked color imaging enhances endoscopic detection of sessile serrated adenoma/polyps. Endosc Int Open 2018;6:E322-E334.

17. Paggi S, Mogavero G, Amato A, et al. Linked color imaging reduces the miss rate of neoplastic lesions in the right colon: a randomized tandem colonoscopy study. Endoscopy 2018;50:396-402.

18. Yoshida N, Inada Y, Yasuda R, et al. Additional thirty seconds observation with linked color imaging improves detection of missed polyps in the right-sided colon. Gastroenterol Res Pract 2018;2018:5059834.

19. Bisschops R, Hassan C, Bhandari P, et al. BASIC (BLI Adenoma Serrated International Classification) classification for colorectal polyp characterization with blue light imaging. Endoscopy 2018;50:211-220.

20. Sano Y, Tanaka S, Kudo SE, et al. Narrow-band imaging (NBI) magnifying endoscopic classification of colorectal tumors proposed by the Japan NBI Expert Team. Dig Endosc 2016;28:526533.

21. Kudo S, Hirota S, Nakajima T, et al. Colorectal tumours and pit pattern. J Clin Pathol 1994;47:880-885.

22. Kanao H, Tanaka S, Oka S, Hirata M, Yoshida S, Chayama K. Narrow-band imaging magnification predicts the histology and invasion depth of colorectal tumors. Gastrointest Endosc 2009;69(3 Pt 2):631-636.

23. Yamashina T, Takeuchi Y, Uedo N, et al. Diagnostic features of sessile serrated adenoma/polyps on magnifying narrow band imaging: a prospective study of diagnostic accuracy. J Gastroenterol Hepatol 2015;30:117-123.

24. Nishihara R, Wu K, Lochhead P, et al. Long-term colorectal-cancer incidence and mortality after lower endoscopy. N Engl J Med 2013;369:1095-1105.

25. Winawer SJ, Zauber AG, Ho MN, et al. Prevention of colorectal cancer by colonoscopic polypectomy: the National Polyp Study Workgroup. N Engl J Med 1993;329:1977-1981.

26. Zauber AG, Winawer SJ, O’Brien MJ, et al. Colonoscopic polypectomy and long-term prevention of colorectal-cancer deaths. $\mathrm{N}$ Engl J Med 2012;366:687-696.

27. Heresbach D, Barrioz T, Lapalus MG, et al. Miss rate for colorectal neoplastic polyps: a prospective multicenter study of back-to-back video colonoscopies. Endoscopy 2008;40:284-290.

28. Rex DK, Cutler CS, Lemmel GT, et al. Colonoscopic miss rates of adenomas determined by back-to-back colonoscopies. Gastroenterology 1997;112:24-28.

29. Kim NH, Jung YS, Jeong WS, et al. Miss rate of colorectal neoplastic polyps and risk factors for missed polyps in consecutive colonoscopies. Intest Res 2017;15:411-418.

30. Lee J, Park SW, Kim YS, et al. Risk factors of missed colorectal lesions after colonoscopy. Medicine (Baltimore) 2017;96:e7468. 